\title{
Development of low-cost photoacoustic imaging systems using very low-energy pulsed laser diodes
}

\author{
Ali Hariri \\ Afreen Fatima \\ Nafiseh Mohammadian \\ Sadreddin Mahmoodkalayeh \\ Mohammad Ali Ansari \\ Nicholas Bely \\ Mohammad R. N. Avanaki
}




\title{
Development of low-cost photoacoustic imaging systems using very low-energy pulsed laser diodes
}

\author{
Ali Hariri, ${ }^{a, b}$ Afreen Fatima, ${ }^{a}$ Nafiseh Mohammadian, ${ }^{a}$ Sadreddin Mahmoodkalayeh, ${ }^{c}$ \\ Mohammad Ali Ansari, ${ }^{c}$ Nicholas Bely, ${ }^{a}$ and Mohammad R. N. Avanaki ${ }^{\mathrm{a}, \mathrm{d}, \mathrm{e}, *}$ \\ ${ }^{a}$ Wayne State University, Bioengineering Department, Detroit, Michigan, United States \\ bUniversity of California, Department of NanoEngineering, San Diego, California, United States \\ 'Shahid Beheshti University, Laser and Plasma Research Institute, Tehran, Iran \\ dWayne State University, School of Medicine, Department Neurology, Detroit, Michigan, United States \\ 'Barbara Ann Karmanos Cancer Institute, Detroit, Michigan, United States
}

\begin{abstract}
With the growing application of photoacoustic imaging (PAI) in medical fields, there is a need to make them more compact, portable, and affordable. Therefore, we designed very low-cost PAl systems by replacing the expensive and sophisticated laser with a very low-energy laser diode. We implemented photoacoustic (PA) microscopy, both reflection and transmission modes, as well as PA computed tomography systems. The images obtained from tissue-mimicking phantoms and biological samples determine the feasibility of using a very lowenergy laser diode in these configurations. @ 2017 Society of Photo-Optical Instrumentation Engineers (SPIE) [DOI: 10.1117/1.JBO.22.7 .075001]
\end{abstract}

Keywords: photoacoustic imaging; photoacoustic microscopy; low-cost diode lasers; reflection mode; transmission mode; photoacoustic computed tomography.

Paper 170008RR received Jan. 10, 2017; accepted for publication Jun. 19, 2017; published online Jul. 11, 2017.

\section{Introduction}

Photoacoustic imaging (PAI) is a promising structural, functional, and molecular imaging technique for a wide range of biomedical applications. PAI combines the technological advances of both optical and acoustic imaging, i.e., the high intrinsic contrast of optical imaging and the spatial resolution of ultrasound imaging. ${ }^{1}$ Every material, including bodily substances, has a specific optical absorption coefficient unique to endogenous chromophores in cells or tissue. The substance to be imaged is illuminated by a nanosecond pulsed laser of a specific wavelength, at which the absorption coefficient of the sample is the highest. Physical characteristics of phantoms and biological tissues and the time scale of energy dissipation within the sample determine the required time scale and the thermal and stress relaxation times over which the light must be delivered.

PAI is a scalable imaging technique, from photoacoustic microscopy (PAM) to photoacoustic computed tomography (PACT). With PAM, a focused light is utilized ${ }^{2}$ to image the vasculature and function of biological tissue $\mathrm{e}^{3,4}$ in more detail $^{5}$ in PACT, a full-field illumination of diffused light is used and deep tissue imaging is possible. Reflection and transmission modes are two detection schemes used in PAM. ${ }^{2,6}$ In reflection mode, the excitation and detection occur on the same side of the sample, while in transmission mode they are on opposite sides. Transmission mode offers a better signal-tonoise ratio (SNR), although the technique is limited to imaging thin tissues, ${ }^{7-9}$ as opposed to reflection mode that can be used for imaging thicker tissues. ${ }^{9-13}$

PAI usually uses solid-state and dye lasers because of their short pulse width and large pulse energy. These lasers are usually bulky and expensive and require high maintenance. On the other hand, diode lasers are compact and inexpensive

*Address all correspondence to: Mohammad R. N. Avanaki, E-mail: mrn. avanaki@wayne.edu and can perform with a high repetition rate. Diode lasers are semiconductor P-N junction diodes that employ current as the pump source. In this study, we use pulsed laser diodes (PLD). PLDs have lower energy per pulse compared to solid-state lasers, e.g., $\mu \mathrm{J}$ or $\mathrm{nJ}$ versus $\mathrm{mJ}$ or $\mathrm{J}^{3,14-16}$ PLDs are available in a wide spectrum of wavelengths, which makes them suitable for photoacoustic (PA) spectroscopy. ${ }^{17}$ They are used in a variety of clinical applications, such as oximetry, ${ }^{18}$ transillumination imaging, ${ }^{19}$ and ophthalmology. ${ }^{20,21}$ PLDs do not require an additional cooling system, which makes them low-maintenance light sources. Because PLDs have low energy, the obtained PA signal from these sources needs to be averaged multiple times to reach to an acceptable SNR. Averaging is a time-consuming procedure, which affects the temporal resolution of the imaging system and prevents them from being used in the applications where a faster imaging system is desired, e.g., hemodynamic monitoring and circulating tumor cell imaging.

Here, we demonstrate the results of low-cost (LC) PAI systems in which a very low-energy PLD with the central wavelength of $905 \mathrm{~nm}$ and output peak power of $6 \mathrm{~W}$ is used. We explain and present the experimental results of three different PAI configurations: transmission mode PAM, reflection mode PAM, and PACT. The images obtained from tissue-mimicking phantoms and biological samples determined the feasibility of using a very low-energy laser diode in these configurations.

\section{Material and Method}

A PLD with the central wavelength of $905 \mathrm{~nm}$ (905D1S03X, Laser Component Co., Bedford, New Hampshire), a peak power of $6 \mathrm{~W}$, and pulse width of $55 \mathrm{~ns}$ operating at maximum repetition rate of $20 \mathrm{KHz}$ is used in the configuration of our PAI systems. The PLD beam is diverging. The large divergent beam is an undesired characteristic of the PLD. To convert the PLD 
Table 1 Comparison of different collimation methods for PLDs.

\begin{tabular}{lccc} 
Collimation & Cost & $\begin{array}{c}\text { Beam } \\
\text { quality }\end{array}$ & $\begin{array}{c}\text { Beam } \\
\text { focusability }\end{array}$ \\
\hline Single aspheric lens & Average & Poor & Good \\
Two cylindrical lenses & High & Fair & Good \\
Aspheric lens + anamorphic prisms & High & Fair & Excellent \\
\hline
\end{tabular}

linear pattern to a tightly focused pattern, several methods have been tested in the literature. The most popular method is to use a single aspheric lens to collimate the PLD. ${ }^{22}$ To focus the PLD beam, a two-lens system is utilized: one lens collimates the highly divergent beam and the second lens focuses the collimated light. Another method for collimating while simultaneously circularizing the beam emitted by a PLD is to use two orthogonally positioned cylindrical lenses. One lens is placed to collimate the beam in the fast axis direction and another in slow axis direction. ${ }^{23}$ A pair of anamorphic prisms is used in addition to an aspheric lens for PLD beam circularization. In this way, a collimated elliptical beam can be circularized by either expanding in the slow axis of the ellipsis or compressing in the fast axis direction. ${ }^{24}$ In Table 1 , a comparison between size, cost, ellipticity, beam quality, and beam focusability of different collimation methods of PLD is given.

Because of the large divergence in the fast axis direction, the lens used to collimate or focus a laser diode beam is a combination of at least one aspheric surface to correct the spherical aberration and a numerical aperture (NA) of at least 0.3 to avoid severe beam truncation. Here, we proposed using an objective lens that includes aspheric lenses with NA equal to 0.6 (40×, Newport Cooperation, California) to focus the beam of the PLD on the sample. The scheme for the focusing optics has been evaluated using ZEMAX 13 with the goal of reaching a $100-\mu \mathrm{m}$ focal point on the sample and efficiently conserving the energy (see Fig. 1). Uniform intensity distribution is

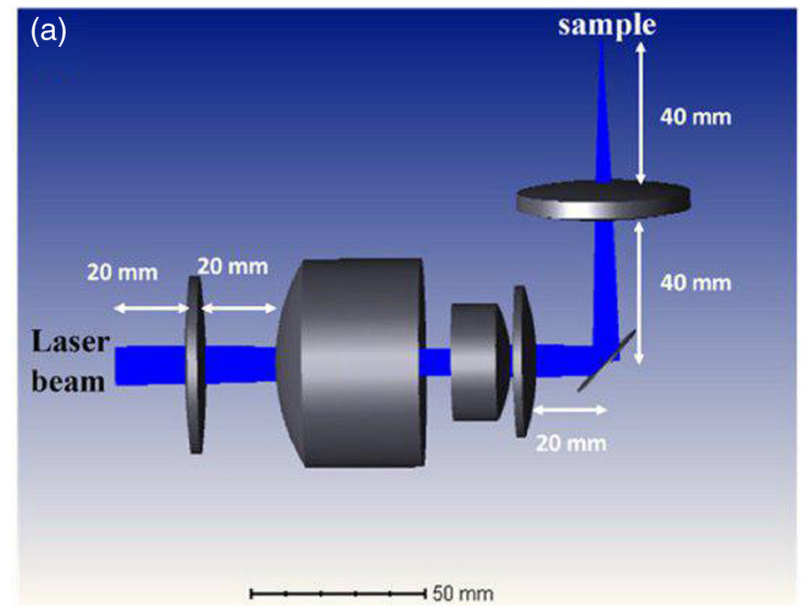

(c)

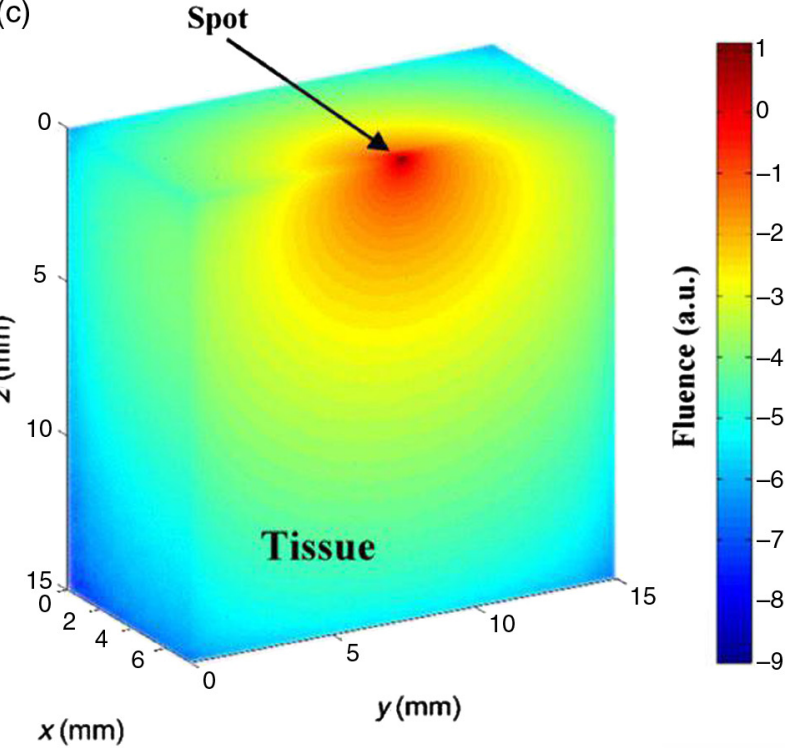

(b)

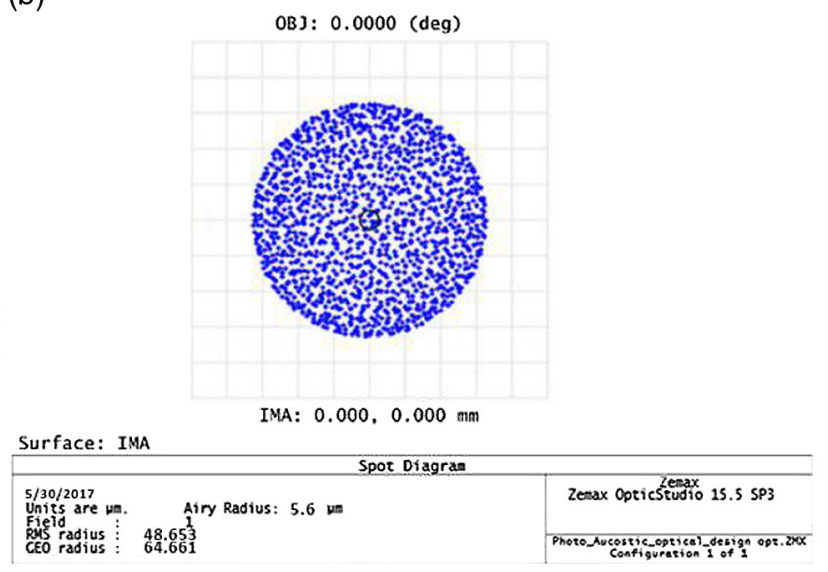

(d)

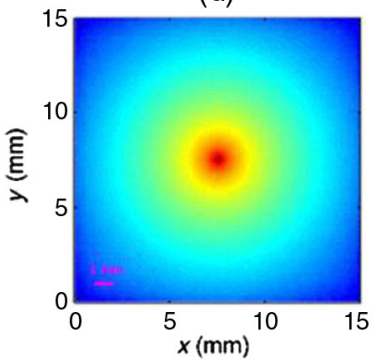

(f)

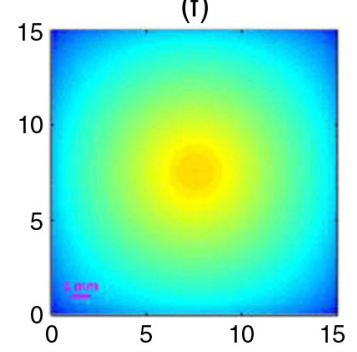

(e)

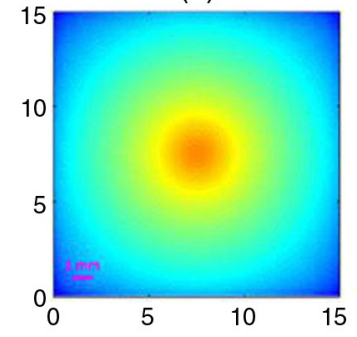

(g)

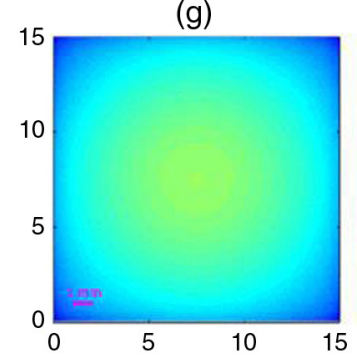

Fig. 1 ZEMAX simulation of the focusing elements: (a) optical components of the focusing scheme, (b) point spread function of the beam on the sample, (c) cross-sectional view of the fluence map (logarithmic scale) for the biological tissue, (d) logarithmic fluence map on the surface, (e) at the depth of $1 \mathrm{~mm}$, (f) at the depth of $2 \mathrm{~mm}$, and (g) at the depth of $5 \mathrm{~mm}$. 


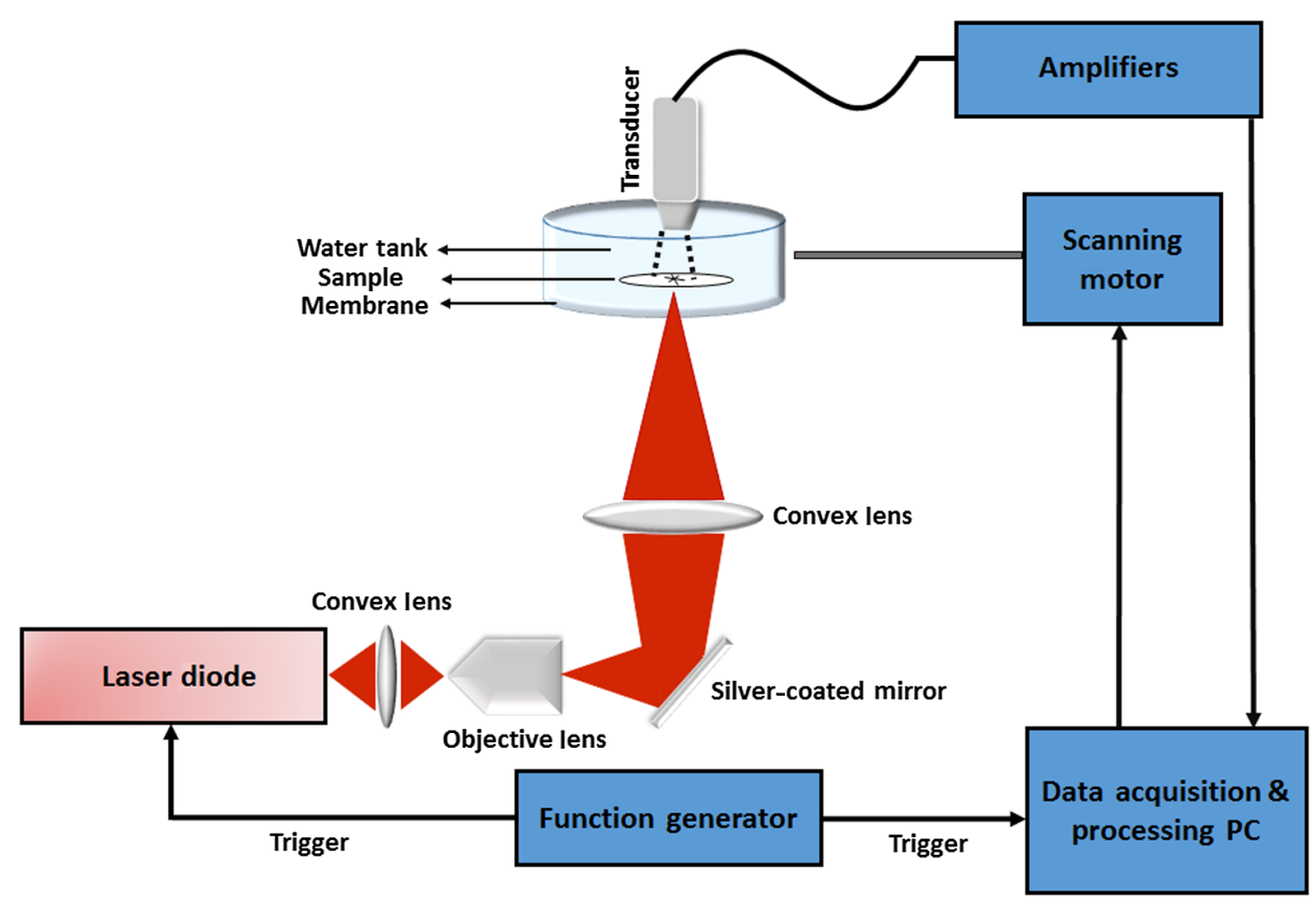

Fig. 2 Schematic of our transmission mode LC-PAM.

considered in this design for a laser beam. Using this optical configuration, a spot size of $97.3 \mu \mathrm{m}$ [Fig. 1(b)] is reached and can be considered the spatial resolution of the PAM system. The working distance of the objective lens is about $600 \mu \mathrm{m}$, and the overall depth of focus is about $300 \mu \mathrm{m}$.

We also showed how 905-nm light is propagated through a tissue. The tissue is considered a single layer $15 \times 15 \times 15 \mathrm{~mm}$ $(L \times W \times H)$ cube with optical properties of $\mu_{\mathrm{a}}=0.35 \mathrm{~cm}^{-1}$ and $\mu_{\mathrm{s}}^{\prime}=18 \mathrm{~cm}^{-1}$, corresponding to that of biological tissue for $905-\mathrm{nm}$ wavelength. ${ }^{25}$ A light source illuminates the surface of the tissue with a spot size of $100 \mu \mathrm{m}$ in diameter [Fig. 1(c)]. Normalized fluence is calculated by Monte Carlo simulation. $2.5 \times 10^{8}$ photons are simulated using MCX software. ${ }^{26}$ The size of voxels is $0.1 \times 0.1 \times 0.1 \mathrm{~mm}$. Figure 1 (c) shows a fluence map (logarithmic scale) at a vertical cross section with $x=7.5 \mathrm{~mm}$, which is where the source is located. Figures $1(\mathrm{~d})-1(\mathrm{~g})$ show the fluence map at the $x$ - $y$ plane on the surface, at the depths of 1,2 , and $5 \mathrm{~mm}$ below the surface, respectively.

In the following, three LC PAI configurations are presented.

\subsection{Transmission Mode Low-Cost Photoacoustic Microscopy}

In transmission mode, the transducer is placed at a 180-deg angle with the incident light perpendicular to the sample (see Fig. 2).

\subsection{Reflection Mode Low-Cost Photoacoustic Microscopy}

In reflection mode PAM, a transducer is held at a 40-deg angle ${ }^{27}$ with the incident light focused on the sample as shown in Fig. 3.
In both configurations, the diverging beam passes through a convex lens (AC254-100-A1, Thorlabs) with a 100-mm focal length and 50-mm diameter to focus the laser beam on the objective lens. An objective lens (40×, Newport Corporation, California) with the NA of 0.65 and a magnification of 40 is used to spherically shape the beam. The beam is then refracted perpendicularly by a mirror and focused onto the imaging target through another convex lens (AC254-060-A1, Thorlabs) with 60-mm focal length and 50-mm diameter. An $X-Y$ stage (ASI LX-4000, Applied Scientific Instrumentation, Inc., Eugene, Oregon) is used for transversal scanning of the sample. The acoustic signal generated at each spot is collected by a stationery cylindrical unfocussed ultrasonic transducer (V312-SU immersion transducers, Olympus). The active element diameter of the transducer is $6 \mathrm{~mm}$ and its central frequency is $10 \mathrm{MHz}$ with $71 \%$ bandwidth. The signal is amplified by passing it through a cascade of three low-noise amplifiers (ZFL-500 Mini Circuits RF/Microwave Components, Brooklyn), therefore, providing a gain of $69.54 \mathrm{~dB}$. The data acquisition is performed using an field-programmable gate array-based National Instrument (NI) system. NI LabVIEW software is used as a platform to acquire signal, analyze it, and generate an image. The sampling frequency in the DAQ card is set to $50 \mathrm{MS} / \mathrm{s}$.

\subsection{Low-Cost Photoacoustic Computed Tomography}

The optical setup is slightly modified for the computed tomography configuration (see Fig. 4). The same PLD is used in the setup. The PLD is placed $3 \mathrm{~cm}$ away from the top of the sample, where the illuminated area on the imaging target is sufficiently large to cover the imaging target. A diffuser (DG10-120- $\varnothing 1$ in. Unmounted N-BK7 Ground Glass Diffuser, 120 Grit, Thorlabs) is placed $5 \mathrm{~mm}$ away from the light source. A $5 \mathrm{~mm} \times 15 \mathrm{~mm}$, thin rectangular piece of black tape is placed in a gel phantom 


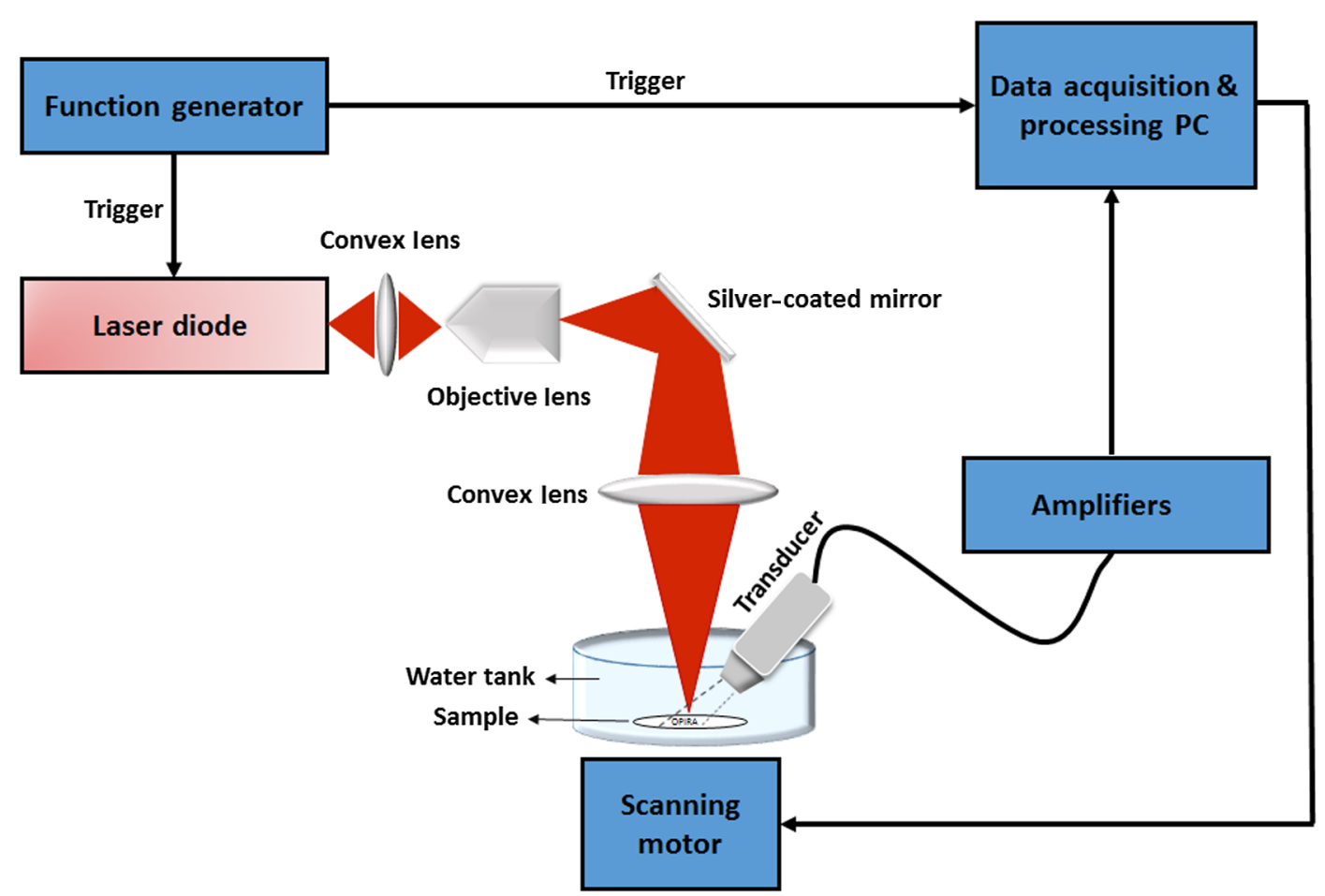

Fig. 3 Schematic of our reflection mode LC-PAM.

comprising of $3 \%$ agar powder in water. We have explained the phantom reconstruction procedure previously. ${ }^{28}$ A cylindrically focused ultrasonic transducer (V326-SU immersion transducers, Olympus) is placed on a cylinder. The transducer is positioned horizontally in the same plane as the black tape. The diameter over which the tomography scanning is performed is $75 \mathrm{~mm}$. The cylinder is rotated using a stepper motor (Applied Motion Products Inc.), the driver of which (ST5-Si-NN, Applied Motion Products Inc.) is controlled by NI LabVIEW software. The transducer is rotated in 120 steps circularly around the phantom, and, at each step, 1000 signals are acquired and averaged. We use a filtered back projection image reconstruction algorithm to generate an image.

For all three configurations, the laser repetition rate is controlled by a function generator (ATTEN ATF20B, OscilloWave Inc.). The function generator is also used to synchronize the data acquisition card and the laser illumination by providing a standard transistor-transistor logic signal.

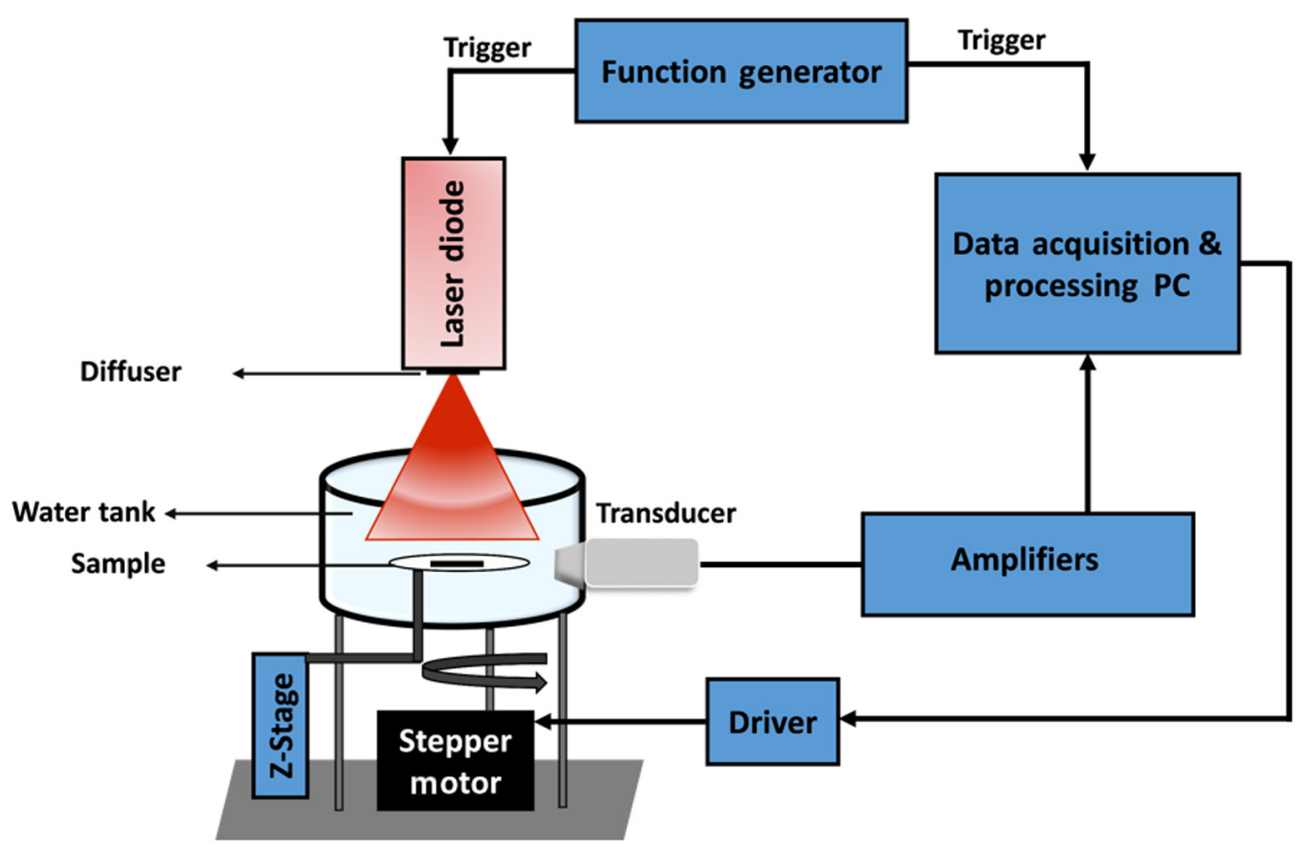

Fig. 4 Schematic of our LC-PACT system. 

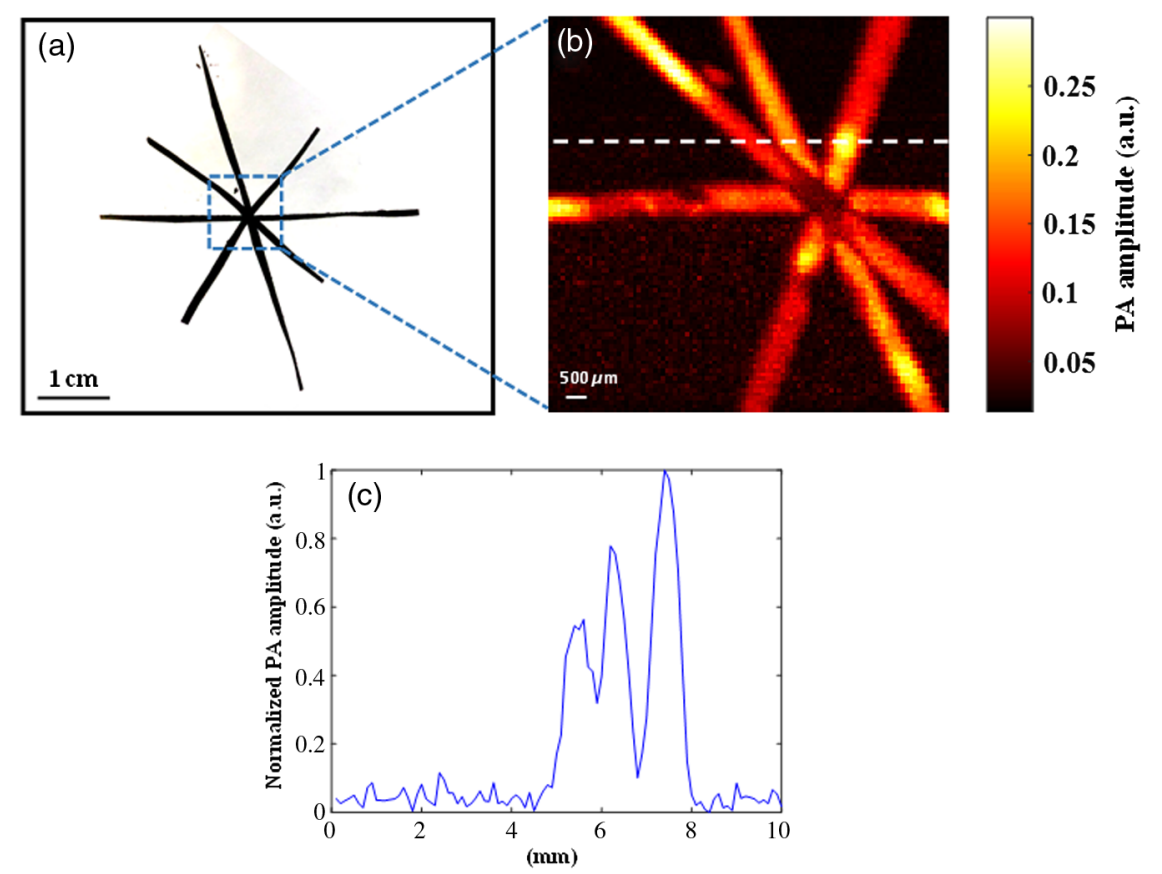

Fig. 5 Transmission mode LC-PAM results: (a) photograph of a star pattern constructed by black tape as an imaging target, (b) PA image of the target with the size of $10 \mathrm{~mm} \times 10 \mathrm{~mm}$, and (c) image intensity profile across the PA image, along the dotted line.

\section{Results}

\subsection{Transmission Mode Low-Cost Photoacoustic Microscopy}

Transmission mode optical resolution-PAM was developed with the setup given in Fig. 2. We imaged a star pattern, constructed of several crossed strips of black tape, for evaluation of the system performance. Two hundred times averaging was used in this experiment. The SNR of the resultant PA signal was measured as $9.5 \mathrm{~dB}$. The sample was scanned by a motorized $X-Y$ scanner with 100 points $(X$-direction $) \times 100$ points $(Y$-direction $)$ and a step size of $100 \mu \mathrm{m}$. Figure 5(a) shows a photograph of the imaging target in a gel phantom. Figure 5(b) shows the PA image of the phantom. In Fig. 5(c), an intensity profile across the PA image, along the dotted line indicated in Fig. 4(b), is shown.

\subsection{Reflection Mode Low-Cost Photoacoustic Microscopy}

The performance of reflection mode LC-PAM is evaluated using several phantoms. The results of two of them are demonstrated in Fig. 6. The phantoms were imaged. One of them was a cross made of black tape (horizontal width $<850 \mu \mathrm{m}$ and vertical
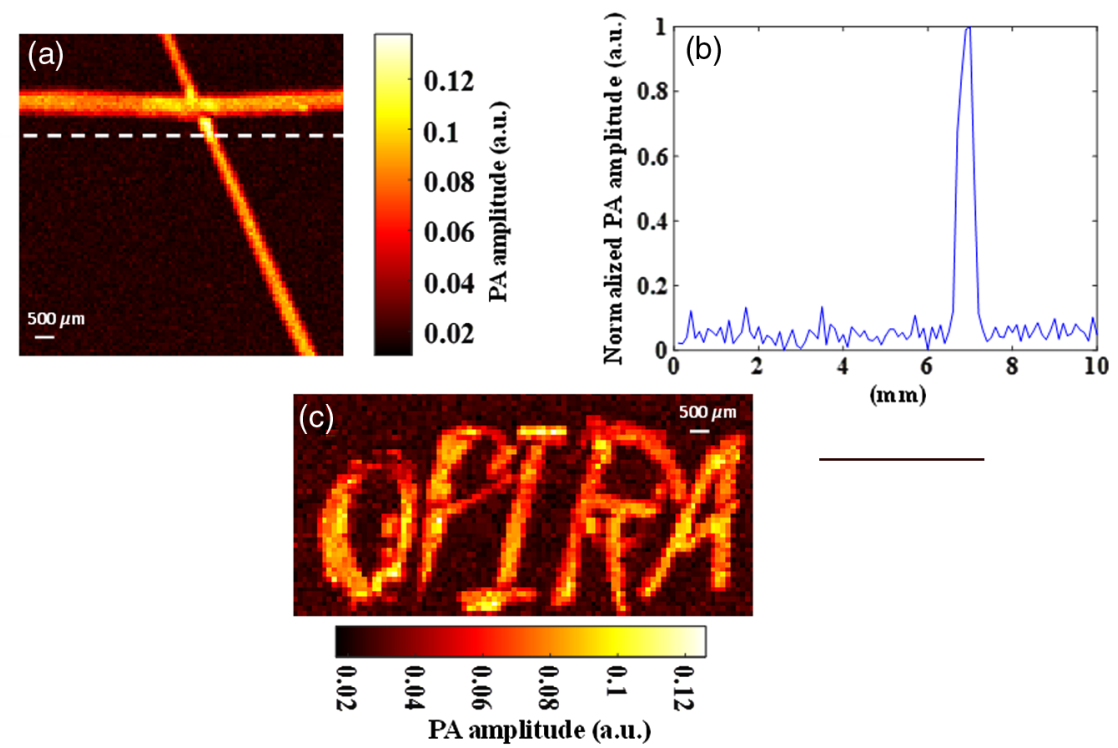

Fig. 6 Reflection mode LC-PAM results: (a) photograph of a cross phantom made with black tape, (b) intensity profile along the dotted line across the PA image in (a), and (c) PA image of the word "OPIRA" made of black tape. 

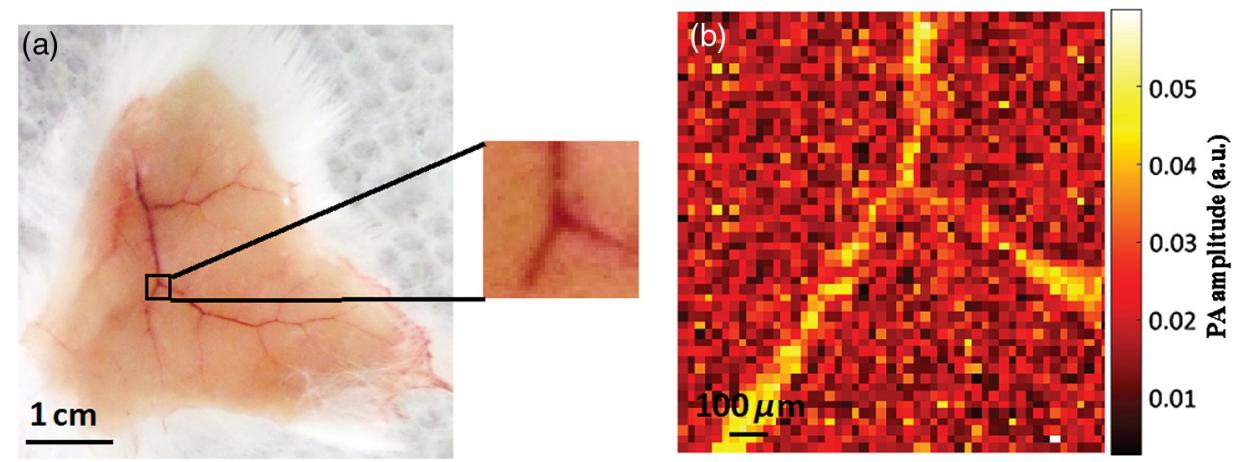

Fig. 7 Reflection mode LC-PAM ex-vivo results: specified and (b) PA image.

width $<400 \mu \mathrm{m}$ ), and another one was the word "OPIRA" (the name of our lab) also made of black tape $(500 \mu \mathrm{m}<$ tape width $<750 \mu \mathrm{m}$ ). In this experiment, the SNR of the PA signal was measured as $6.5 \mathrm{~dB}$. We used 500 times averaging of the PA signals to form a meaningful PA image. Figures 6(a) and 6(b) show photographs of the phantoms. Figure 6(a) shows the PA images of the phantoms. In Fig. 6(b), an intensity profile across the PA image, along the dotted line indicated in Fig. 6(a), is shown. The samples are scanned by a motorized $X-Y$ scanner with 100 points $\times 100$ points and a step size of $100 \mu \mathrm{m}$. In Fig. 6(c), we cropped half of the image that did not contain any absorber.

We also imaged a mouse skin ex-vivo, using reflection mode LC-PAM. The sample was placed on a white nonabsorbing material in a water tank. The SNR of the PA signal obtained from the sample after 5000 times averaging was $2 \mathrm{~dB}$. Figure 7(a) is a photograph of the mouse skin with the region that was imaged by reflection mode LC-PAM. Figure 7(b) shows the PA image of the vasculature in the mouse skin. The number of scanning points used in this experiment was 50 points $(X$-direction $) \times 50$ points $(Y$-direction $)$ with a step size of $100 \mu \mathrm{m}$. Acquisition time was about $12 \mathrm{~min}$. Wayne State University Animal Care and Use Committee approved the animal tissue imaging study.

\subsection{Low-Cost Photoacoustic Computed Tomography}

The imaging target here was a thin strip of black tape. The PA image was constructed when each acquisition was repeated
1000 times and averaged. The SNR of the resultant PA signals for LC-PACT configuration was measured as $5.4 \mathrm{~dB}$. The image is generated from 120 view angles; each view was taken at 7.2-deg increments. Figure 8 shows the reconstructed PACT image using a filtered back projection algorithm. Acquisition time, including the reconstruction processes, was about $30 \mathrm{~s}$ for 120 view angles.

\section{Discussion}

We demonstrated the results of using a very low-energy PLD in PAI systems. Pulse width and pulse energy are the two main limitations of most low-energy light sources. ${ }^{28-30}$ The large pulse width limits the stress confinement and affects the acoustic wave generated from small vessels. ${ }^{16}$ Low pulse energy light sources generate low SNR PA signals with limited penetration depth. To enhance the SNR, a large number of averaging is used. This, however, affects the temporal resolution of the system. We devised some signal enhancement algorithms similar to averaging but more efficient, i.e., achieving the same SNR with a much smaller number of averaging. Therefore, the very LC of the low-energy laser source is advantageous; the spatial and temporal resolutions of the images produced may not be satisfactory for some applications.

In this paper, we showed the performance of three configurations of LC PAI using LC PLDs: transmission mode LC-PAM, reflection mode LC-PAM, and LC-PACT. The PA images of several tissue-mimicking phantoms and biological samples were shown. Several challenges were tackled in this study. The main challenge of the microscopy configurations was to (a)

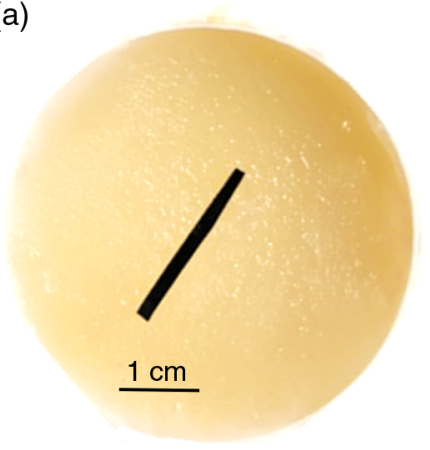

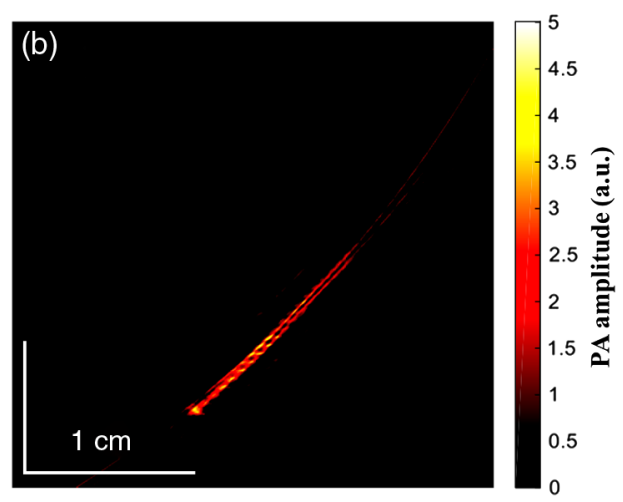

Fig. 8 Evaluation of the performance of the LC-PACT with 120 view angles. The imaging target is a thin black tape. 
focus the diverging linear beam to a tightly spherical shape (Fig. 1). Several configurations were suggested in the literature $^{16}$ to solve this problem. We chose an optical setup in which the laser energy on the sample is maximum (tightest focus and highest energy per pulse). This configuration was simulated in ZEMAX. Although we reported both transmission mode and reflection modes, the SNR of the PA signal in transmission mode was much higher. Transmission mode configuration, however, has limited applications, e.g., imaging of a very thin tissues ex-vivo. To the best of our knowledge, this study demonstrated, for the first time, the development of a reflection mode LC-PAM with a very low-energy PLD for imaging biological phantoms (Fig. 7). In reflection mode LC-PAM configuration, the transducer was tilted $40 \mathrm{deg}$, which yielded a lower SNR. We believe that the results will improve if the location of the transducer to the sample is iteratively tested. There are several factors that affect the quality of the resultant images. The pulse width of the PLD was about $55 \mathrm{~ns}$, which limits the stress confinement and affects the acoustic wave generated from small vessels. In-vivo imaging using LC-PACT is the future plan for this study.

The low excitation energy is another major restriction of using low-energy PLDs. By averaging numerous PA signals at each position, the SNR can be increased. We are developing signal processing algorithms to reduce the number of averaging. ${ }^{31}$ This will make the implementation of such systems faster and hence more practical.

\subsection{Image Formation Time}

Image formation time highly depends on the PLD repetition rate and the number of averaging we consider at each pixel location. Since the number of averaging is different for different configurations, it is calculated for each setup separately.

a. Transmission mode LC-PAM: For a region of interest (ROI) size of $100 \times 100$ pixels, to form an LC-PAM image in the transmission mode, 10,000 acquisitions with 200 repetitions (averaging) for each signal is required. Hence, the total acquired signal is $10,000 \times$ $200=2,000,000$. With the laser repetition rate of $20 \mathrm{KHz}$, each signal takes about $0.05 \mathrm{~ms}$ (accounting for arming time of the analog-to-digital converter module and writing the data on the hard drive); the time required to form an image is $2,000,000 \times$ $0.05 \mathrm{~ms}=100 \mathrm{~s}$. Considering another $20 \mathrm{~s}$ for initialization of the LabVIEW program, the acquisition time per image is about $2 \mathrm{~min}$.

b. Reflection mode LC-PAM: The calculation to form an image in reflection mode LC-PAM is similar to that of transmission mode LC-PAM, with the difference that 500 repetitions (averaging) are required due to the weaker signal. Thus, the time required to form an image is $5,000,000 \times 0.05 \mathrm{~ms}=250 \mathrm{~s}$. Considering another $20 \mathrm{~s}$ for initialization of the LabVIEW program, the acquisition time per image is about $5 \mathrm{~min}$.

c. LC-PACT: With this configuration, a finer temporal resolution is achieved. Acquisition time, including the reconstruction process, is about $30 \mathrm{~s}$. The acquisition time is increased when a greater number of view angles is used.

\subsection{Spatial Resolution}

In PAM configurations (both transmission and reflection modes), the beam size determines the spatial resolution. Based on our ZEMAX simulation results demonstrated in Fig. 1 and confirmed in the experiments, the spatial resolution was measured to be about $100 \mu \mathrm{m}$ [Fig. 1(b)]. We also measured the lateral resolution of both PAMs experimentally by imaging the edge of a sharp blade and a carbon fiber with a step size of $1 \mu \mathrm{m}$. The edge spread function was fitted using the maximum amplitude projection data across a line. The first derivative of the edge spread function creates a line spread function, and the full width at half maximum of that was considered the lateral resolution. The measured lateral resolution was $108 \mu \mathrm{m}$ for the transmission mode LC-PAM and $112 \mu \mathrm{m}$ for reflection mode LC-PAM. For PACT, the spatial resolution depends on the number of view angles and the reconstruction algorithm. In our study with 120 view angles and the back projection reconstruction algorithm, the spatial resolution measured $155 \mu \mathrm{m}$.

\section{Conclusion}

In this study, we have presented the feasibility of using a very low-energy PLD ( $\sim 6 \mathrm{~W})$ in the configuration of PAI systems. We have demonstrated the use of such LC laser sources with three PA systems: transmission mode LC-PAM, reflection mode LC-PAM, and LC-PACT. The results of imaging several tissue-mimicking phantoms and mouse skin vasculature showed that, using such very low-energy lasers, we will be able to build compact PAI systems. The current drawback of such systems is their coarse temporal resolution. We are developing signal processing techniques that decrease the number of repetitions to 100 times or lower, which significantly improves the temporal resolution of such LC PAI systems.

\section{Disclosures}

The authors have no relevant financial interests in the paper and no other potential conflicts of interest to disclose.

\section{Acknowledgments}

The authors thank engineers from Laser Components Co. for their helpful discussion. The authors acknowledge the Wayne State University Startup Fund.

\section{References}

1. S. Hu, K. Maslov, and L. V. Wang, "Three-dimensional optical-resolution photoacoustic microscopy," J. Visualized Exp. 51, e2729 (2011).

2. K. Maslov et al., "Optical-resolution photoacoustic microscopy for in vivo imaging of single capillaries," Opt. Lett. 33(9), 929-931 (2008).

3. T. J. Allen and P. C. Beard, "Pulsed near-infrared laser diode excitation system for biomedical photoacoustic imaging," Opt. Lett. 31(23), 34623464 (2006).

4. R. G. Kolkman, W. Steenbergen, and T. G. van Leeuwen, "In vivo photoacoustic imaging of blood vessels with a pulsed laser diode," Lasers Med. Sci. 21(3), 134-139 (2006).

5. L. V. Wang and S. Hu, "Photoacoustic tomography: in vivo imaging from organelles to organs," Science 335(6075), 1458-1462 (2012).

6. S. Hu and L. V. Wang, "Optical-resolution photoacoustic microscopy: auscultation of biological systems at the cellular level," Biophys. J. 105(4), 841-847 (2013).

7. C. Zhang, K. Maslov, and L. V. Wang, "Subwavelength-resolution label-free photoacoustic microscopy of optical absorption in vivo," Opt. Lett. 35(19), 3195-3197 (2010). 
8. Y. Yuan, S. Yang, and D. Xing, "Optical-resolution photoacoustic microscopy based on two-dimensional scanning galvanometer," Appl. Phys. Lett. 100(2), 023702 (2012).

9. W. Song et al., "Reflection-mode in vivo photoacoustic microscopy with subwavelength lateral resolution," Biomed. Opt. Express 5(12), 4235-4241 (2014).

10. J. Chen et al., "Blind-deconvolution optical-resolution photoacoustic microscopy in vivo," Opt. Express 21(6), 7316-7327 (2013).

11. C. Zhang et al., "Reflection-mode submicron-resolution in vivo photoacoustic microscopy," J. Biomed. Opt. 17(2), 020501 (2012).

12. K. H. Song and L. V. Wang, "Deep reflection-mode photoacoustic imaging of biological tissue," J. Biomed. Opt. 12(6), 060503 (2007).

13. R. G. Kolkman, W. Steenbergen, and T. G. van Leeuwen, "Reflection mode photoacoustic measurement of speed of sound," Opt. Express 15(6), 3291-3300 (2007).

14. L. Zeng et al., "3D-visual laser-diode-based photoacoustic imaging," Opt. Express 20(2), 1237-1246 (2012).

15. P. LeBoulluec, H. Liu, and B. Yuan, "A cost-efficient frequency-domain photoacoustic imaging system," Am. J. Phys. 81(9), 712-717 (2013).

16. T. Wang et al., "A low-cost photoacoustic microscopy system with a laser diode excitation," Biomed. Opt. Express 5(9), 3053-3058 (2014).

17. T. J. Allen and P. C. Beard, "High power visible light emitting diodes as pulsed excitation sources for biomedical photoacoustics," Biomed. Opt. Express 7(4), 1260-1270 (2016).

18. D. M. Shemwell and G. R. Ryan, "Method and apparatus for diode laser pulse oximetry using fiber optical cables," U.S. Patent 6,026,312 (2000).

19. R. Berg, O. Jarlman, and S. Svanberg, "Medical transillumination imaging using short-pulse diode lasers," Appl. Opt. 32(4), 574-579 (1993).

20. C. Moorman and A. Hamilton, "Clinical applications of the MicroPulse diode laser," Eye 13(2), 145-150 (1999).

21. P. K. Upputuri and M. Pramanik, "Pulsed laser diode based optoacoustic imaging of biological tissues," Biomed. Phys. Eng. Express 1(4), 045010 (2015).

22. J. H. Jung and Y. J. Choi, "Aspheric lens for simultaneously collimating and shaping a laser diode beam," U.S. Patent 5,572,367 (1996).

23. K. Roth et al., "Imaging of a linear diode bar for an optical cell stretcher," Biomed. Opt. Express 6(3), 807-814 (2015).

24. T. Kasuya, T. Suzuki, and K. Shimoda, "A prism anamorphic system for Gaussian beam expander," Appl. Phys. 17(2), 131-136 (1978).

25. A. Bashkatov et al., "Optical properties of human skin, subcutaneous and mucous tissues in the wavelength range from 400 to $2000 \mathrm{~nm}$," J. Phys. D: Appl. Phys. 38(15), 2543-2555 (2005).

26. Q. Fang and D. A. Boas, "Monte Carlo simulation of photon migration in 3D turbid media accelerated by graphics processing units," Opt. Express 17(22), 20178-20190 (2009).

27. Z. Xie et al., "Laser-scanning optical-resolution photoacoustic microscopy," Opt. Lett. 34(12), 1771-1773 (2009).

28. A. Hariri et al., "Low cost photoacoustic spectroscopy system for evaluation of skin health," Proc. SPIE 9976, 99760X (2016).

29. P. K. Upputuri and M. Pramanik, "Performance characterization of low-cost, high- speed, portable pulsed laser diode photoacoustic tomography (PLD-PAT) system," Biomed. Opt. Express 6(10), 4118-4129 (2015).

30. A. Hariri, A. Fatima, and M. Nasiriavanaki, "A cost-effective functional connectivity photoacoustic tomography (fcPAT) of the mouse brain," Proc. SPIE 10064, 1006439 (2017).

31. A. Hariri et al., "Photoacoustic signal enhancement: towards utilization of very low-cost laser diodes in photoacoustic imaging," Proc. SPIE 10064, 100645L (2017).
Ali Hariri is a PhD student in Nanoengineering Department at the University of California-San Diego. He started his $\mathrm{PhD}$ in Biomedical Engineering Department at Wayne State University in 2015 as a graduate research assistant in OPIRA lab. He received his master's degree and bachelor's degree in Biomedical Engineering from Sharif University of Technology and Amirkabir University of Technology in Iran. He worked on developing different configurations of photoacoustic imaging technique including computed tomography and microscopy (both acoustic and optical resolution).

Afreen Fatima is a graduate student in Biomedical Engineering at Wayne State University and holds a bachelor's degree in electronics and communication engineering from Osmania University. She has been working as a student research assistant in OPIRA lab since September 2015, with research specialization in instrumentation. Her research so far has been mainly on the design and development of low cost, efficient, portable and point of care photoacoustic imaging systems. Currently, she is a researcher in the Biomedical Engineering Department. Her focus is in electronics, programming, optics and acoustic technology.

Nafiseh Mohammadian received her BSc degree in physics from the University of Isfahan and her MSc degree in electro-optical engineering from the University of Technology in Iran. In 2015, she joined OPIRA lab as a research scholar at Wayne State University. She worked on increasing the imaging depth in optical imaging methods by suppressing the turbidity that exists in biological tissues using spatial light modulator. Currently, she is a PhD student in CREOL working on complex coherence measurement for imaging in Ayman Abouraddy's research group.

Sadreddin Mahmoodkalayeh received his MSc degree in physics from Sharif University of Technology, Tehran, Iran, in 2013. Since then, he has been a PhD student of physics at Shahid Beheshti University, Tehran, Iran. Currently, his research is in the field of bioimaging including diffuse optical tomography and photoacoustic imaging.

Mohammad Ali Ansari received his MSc and PhD degrees in photonics from Shahid Beheshti Univertity, Tehran, Iran, in 2006 and 2010, respectively. Since then, he had been a faculty member of Laser and Plasma Research Institute (LAPRI), at Shahid Beheshti University. He is the head of Optical Bio-imaging Lab with main research areas including functional near-infrared spectroscopy and diffuse optical tomography.

Nicholas Bely is undergraduate student in OPIRA lab at Wayne State University. His focus is mainly on developing Labview programs for different photoacoustic imaging configurations (tomography and microscopy).

Mohammad R. N. Avanaki received a PhD with outstanding achievement in medical optical imaging and computing from the University of Kent, United Kingdom, in 2012. His bachelor's and master's degrees with honors are in electronics engineering. In 2014, he completed a three-year postdoctoral fellowship at Washington University in St. Louis, in the OILab. He is currently an assistant professor in the Biomedical Engineering, Dermatology and Neurology Departments of Wayne State University and Scientific member of Karmanos Cancer Institute. He is also serving as the chair of bioinstrumentation track. 